\title{
Disscusion on the Unloading - Seepage Coupled Model of Damaged Rock
}

\author{
ZHAO Erping ${ }^{1, a}$, LU Bo ${ }^{1, b}$, JIA Xiaobing ${ }^{2, c}$ \\ ${ }^{1}$ Key Laboratory of Geological Hazards on Three Gorges Reservoir Area(China Three Gorges \\ University),Ministry of Education, Yichang \\ ${ }^{2}$ Three Gorges Vocational College of Electric Power, Yichang \\ a58517507@qq.com, ${ }^{\mathrm{b}}$ 1130354182@qq.com, ${ }^{\mathrm{c} x \mathrm{x} \_j \mathrm{xb} @ 163 . c o m}$
}

Keywords: unloading, damaged rock, stress-seepage coupling, unloading-seepage coupling Abstract. It is common to encounter the excavation unloading of rock mass in the actual practice of construction work for tunnels, underground powerhouse, dams and mining, etc. The damage and fracture of the internal structure as well as the change of seepage for rock due to the excavation and unloading of rock mass constitute a major factor that causes engineering instability or geological hazards. Though analyzing the existing seepage-stress coupled model, the paper gives the research prospect and direction of the unloading-seepage coupled model of damaged rock.

\section{Introduction}

Natural rock is in a certain stress state, the natural causes or human engineering activities will break the balance of the original rock stress field, resulting in the damage and rupture of the internal structure of rock mass, degrading the mechanical properties and improving the permeability characteristics of rock mass. Therefore, the migration characteristics of water changes. Malpasset dam burst when the first impoundment (in French,1959), instability sliding occurred in the slope of Three Gorges Reservoir when the reservoir water level raised and fell. Water inrush accidents occurred in the process of mining, all of which are closely related to the groundwater seepage characteristics. The damage and fracture of internal structure in rock mass caused by engineering disturbances and the change of rock mass's seepage characteristic is one of the main causes that lead to instability of engineering and geological disasters. Therefore, it is very important to research the relationship between seepage and stress in the damaged rock mass.

In practical engineering practices, such as tunnels, underground powerhouses, dams and mining, and so on, often encounter excavation of rock mass and soil. Rock mass's excavation and unloading cause rock stress state changing, making rock mass's quality deteriorating, and leading to the generation and expansion of fissure in rock mass. Crack generated provide convenience to water infiltration. At the same time, the water infiltration, which in turn affects the stress field of rock mass, changing the internal structure of rock mass. Under the combined action of rock mass unloading, water seepage and rock mass stress, the instability of engineering and geological hazards occurred.

Due to the need of engineering construction, the excavation and unloading of rock mass is inevitable; due to the limitation of natural conditions, water seepage in the excavation of rock mass also is inextricably. Therefore, there is an urgent need to carry out the researches on the unloading - seepage coupled effect of damaged rock mass, in order to provide theoretical basis for the safe operation of engineering structure and prevention of the geological disasters.

\section{Analysis of the Current Researches on the Stress-Seepage Coupled Effect of Rock Mass}

Prior to 1960s, the study on seepage effect in rock mass was less. In 1959, French Malpasset dam burst during the first reservoir impounding, Italian Vaiont reservoir landslide in 1963, then the majority of scholars began to pay attention to the hydraulic properties of the rock mass, and began in-depth study of rock mass's hydraulics properties.

Studies on the seepage-stress coupled effect of rock mass, mainly divided into two categories, one is experimental method, through the fitting of the experimental data, and directly obtained the 
relationship between the permeability coefficient and stress. Another kind is based on various mechanical theory and coupling theory, through theoretical derivation, acquired the basic model of stress and seepage coupling, and then use the model to simulate the experimental data and demonstrate the model's rationality. The main research results of seepage - stress coupling model are as follows:

The formula[1] for Snow (1966) is:

$$
K_{e}=K_{0}+A\left(\frac{g e^{2}}{4 v l}\right) \frac{\sigma_{n e}-\sigma_{n 0}}{k_{n}} .
$$

where $K_{e}$ is the fracture permeability coefficient when the effective normal stress is $\sigma_{n e} ; K_{0}$ is the initial permeability coefficient when the initial stress is $\sigma_{n 0} ; \mathrm{V}$ is the coefficient of kinematic viscosity of water; $e$ is gap width; $l$ is fracture spacing; $k_{n}$ is the normal stiffness of fracture; $A$ is the empirical coefficient.

According to a dam pumping test[2,3], Louis (1974) gave the empirical formula between permeability coefficient and stress:

$$
K_{e}=K_{0}+A\left(\frac{g e^{2}}{4 v l}\right) \frac{\sigma_{n e}-\sigma_{n 0}}{k_{n}}
$$

where $k_{0}$ is the initial permeability coefficient, $\sigma$ is normal stress, is the parameter.

Jones (1975) put forward the experience formula of permeability coefficient of calcium carbonate rock fracture:

$$
k_{e}=k_{0} \lg ^{3}\left(\sigma_{n h} / \sigma_{n e}\right) .
$$

Where $\sigma_{n h}$ is the effective normal stress when $k_{e}$ is zero.

Manfredini (1978) gave the relationship[4] between permeability coefficient of isotropic medium and anisotropic medium and average stress of rock mass. After that, Noorishad (1983) put forward the coupling problem[5] of seepage and stress. In 1986, the relationship between the seepage and deformation of rock mass was represented uniformly by the fracture geometry of Oda, and the relationship between the stress and strain of the equivalent medium model was discussed[6].

Iwai (1984) derived the relationship between the crack permeability coefficient and the stress:

$$
k_{e}=k_{0} \frac{1}{\left[1+A\left(\sigma_{n e} / \xi\right)^{t}\right]^{3}} .
$$

where $A, \xi$ and $t$ are the empirical parameters obtained from experiments.

The relationship between the permeability coefficient and the stress derived from Barton (1985) [7]:

$$
k_{e}=k_{0}\left(1+\frac{\sigma_{n e}}{e_{m_{0}} k_{n_{0}}}\right)
$$

The relationship between stress and seepage is established by Erichsen (1987). Sun Guangzhong, Liu Jishan, and so on, derived the relationship between the permeability coefficient and the stress [8] :

$$
k_{e}=k_{0} \exp \left(-\frac{2 \sigma_{n e}}{e_{m_{0}} k_{n_{0}}}\right)
$$


Chai Junrui and Wu Yanqing (1997) put forward the mathematical model of continuous medium which is the coupling analysis of stress field and seepage field [9]. Wu Yanqing and Chai Junrui (2000) proposed a mathematical model of analysis of 3-D model of coupled seepage and stress fields in fracture network of rock mass [10]. In 1997, based on summing up the results of previous studies, Wu Yanqing put forward viewpoints of rock mass hydraulics, proposed the lumped parameter model of the coupling seepage field and stress field, continuous medium model, the equivalent continuous medium model, the fracture network model and double medium model [11,12].

Zheng Shaohe(1999) revealed the relationship between the fracture permeability coefficient and equivalent normal stress under the three-dimensional stress through the natural fracture seepage experiment [13]. LI Xinping (2006) refitted the test results of Zheng Shaohe and modified the equivalent normal stress formula of the fitted formulas [14].

Chai Junrui (2001) proposed equivalent continuous seepage field and stress field coupled model based on the mathematical model of seepage field under the influence of the equivalent continuous rock stress field and the stress field mathematical model under the influence of seepage field [15]. Zhang Dianji built (2003) the single fractured rock mass's permeability coefficient expression considering the dimensional stress and pore water pressure [16]. Liang Bing (2004) deduced stress field and seepage field coupled equations according to the fluid-solid interaction theory, stress field equation and seepage field equation [17]. Guo Yulong (2005) deduced the relation between the permeability coefficient and stress according to the relationship between the normal deformation of the single fracture surface and stress, and the cubic law [18]. Li Yan (2006) established the coupled model of stress and seepage in soft rock, which is based on anisotropic elastic plasticity theory, water balance principle and the nonlinear coupled relation of permeability coefficient [19]. Zhang Jincai and Wang Jianxue (2006) derived the relation between stress and permeability coefficient by using pore elastic mechanics theory [20]. Wang Yuan (2007) established the seepage and stress dynamic coupled equation of the fractured rock mass [21]. Li Yan (2009) carried out the three axial compression tests and seepage tests on the muddy silty sandstone and mudstone, and established the coupled relationship between permeability tensor and stress in porous media according to the case that the principal axis of the rock mass is not consistent with the seepage principal axis. According to the comparison of theoretical and experimental results, they found the argillaceous siltstone and mudstone are consistent with the seepage model of porous media [22]. Zhang Yong (2010) gave the relationship between the permeability coefficient and stress according to the classical basic equation of seepage coupled theory [23]. In 2011, according to the theory of porous media seepage mechanics and the theory of elastic plastic mechanics, Ji Youjun established the coupled mathematical model of stress and seepage [24]. Lin Peng (2013) established the relationship between the permeability coefficient and stress of the equivalent continuum fractured rock mass and the discrete fractured rock mass, and thus analyzed the deformation stability of the Xiluodu arch dam under the coupled action of seepage and stress [25].

\section{Unloading-Seepage Coupling Research Status}

A large number of experiments show that the mechanical properties of rock mass under loading and unloading conditions are very different. Ha Qiuling first proposed the concept of unloading rock mass mechanics from the practice of the Three Gorges project. Afterwards ,many domestic and foreign scholars studied different kinds of rock mass unloading mechanical characteristic test under different unloading methods and theoretical aspects, and the achievements are very rich, they confirmed the unloading mechanics characteristic of the rock mass and loading mechanics characteristic of rock mass are different.

In the excavation of rock mass project, the formation of unloading effect to the rock mass affected the nature of water in the rock mass. During the operation of the reservoir, the depression of water level also formed unloading effect on the slope, thereby affecting the stability of the slope. Under the action of seepage, the mechanical properties and loading conditions of the rock mass after unloading damage are different, and many engineering problems and geological disasters happen because of 
unloading rock mass and seepage coupled action. Therefore, it is very important to carry out the research on the coupled effect of unloading - seepage.

At present, the main results of study on the coupled characteristics of unloading-seepage in rock mass are as follows: in 1984, Kelssal analyzed the change and its reasons of the permeability coefficient of the underground cavern after excavating [26]. In 1996, the relationship between unloading and seepage of the rock mass was preliminarily discussed by Chen Hongkai. In 2001, based on the different theories and empirical formulas, Xu Guangxiang deduced the relationship between the permeability coefficient and the stress when unloading. One is established on the relationship between stress and permeability by Louis, one is based on Bandis's fracture deformation curve and Barton's hydraulic fracture width formula, another is built on the relationship between the permeability coefficient and the stress of rock mass [27]. In 2005, Liang Ninghui and Ai Wanmin deduced the relation between unloading amount and permeability coefficient by the unloading-seepage test of the fractured rock mass. The methods are according to the cubic law and the relationship between stress and closure proposed by Goodman which fitted the test data well [28,29]. In 2016, Wang Wei and Xu Weiya conducted the experimental research on the permeability characteristics of granitic gneiss in different stress path, and qualitatively analyzed the influence of loading and cyclic loading and unloading on permeability of granitic gneiss. The results show that when the osmotic pressure is constant, the permeability of low confining pressure mutates earlier than high confining pressure's. Under the same confining pressure, increasing seepage pressure brings more obvious influence on the permeability increasing section, and with the increase of osmotic pressure, the mutation point of permeability is relatively earlier [30].

\section{Discussion}

Through the analysis on the results of the above research, it is found that the seepage-stress coupled analysis is quite abundant, but the content of the study on the coupling characteristics of the unloading - seepage is needed to be improved.

The results of coupled analysis of unloading-seepage in rock mass can be applied to the stability analysis of foundation excavation, cavern excavation, slope stability and so on. Therefore, it is very important to carry out the coupled study of unloading-seepage in rock mass. It is suggested that by coupled test of unloading-seepage, water absorption test and ultrasonic testing methods, to comprehensively tested the situation of damage and disruption in the rock, and in order to seek the inevitable link between the macroscopic law of the coupled unloading -seepage in rock mass and characteristics of changes in the internal structure, so as to establish the unloading-seepage coupled model of rock mass.

\section{Acknowledgements}

This work was financially supported by the Hubei Natural Science Foundation (2015CFC831) and Key Laboratory of Geological Hazards on Three Gorges Reservoir Area(China Three Gorges University),Ministry of Education (2015KDZ17).

\section{References}

[1] ZHOU Weiyuan. Advanced Rock Mechanics[M]. Beijing: Water Resources and Electric Power Press, 1993.6, p272-295.

[2] Louis C, Maini YNT. Determination of in situ hydranlic paramaters in jointed rock. Rroc 2nd congr ISRM,1970, Vol 1, p235-245.

[3] Louis C. Introduction a hadraulique desroches Orleans, Bureau Research Geologique Miniers, 1974. 
[4] Guo Xuemang. Deformation,Stability and Seepage Problem of Rock Masses as well as Their Interaction[D]. Dalian: Dalian University of Technology, 1990.3.

[5] Atouahi MS Noorishad etal. Stress-fluid flow analysis of fractured rock J.Eng.Mech,1983,10(9):1-13.

[6] Oda,M. An equivalent continuum model for coupled stress and fluid flow ananlysis in jointed rock masses, Water research,1986,13(22):1845-1856.

[7] Barton,N.,Bandis,S.,Bckhtar,K.,Strength, Deformation and Conductivity Coupling of Rock Joints,Int.J.Rock Mech.Min.Sci. \& Geomech.Abstr., Vol.22,No.3,1985,p121-140.

[8] Liu Jishan. The coupled relationships between mechanical and hydraulic parameters of a structural surface and their applications[J]. Hydrogeology \& Engineering Geology, 1988, 02, p7-12.

[9] Chai Junrui, Wu Yanqing. Research on Mathematical Model for Coupled Stress and Seepage Field in Single-Zone Embankment Dam[J]. Journal of Shaanxiwater power, 1997, 13(3):4-7.

[10] Wu Yanqing, Chai Junrui. Analysis of 3-D Model of Coupled Seepage and Stress Fields in Fracture Network of Rock Mass[J]. Journal of Xi'an University of Technology, 2000, 16(1): 1-5.

[11] WuYanqing, ZHANG Zhouyuan. An introduction of rock mass hydraulics [M].Chengdu: Southwest Jiaotong University Press, 1995.

[12] Wu Yanqing. A brief decription ofrock masshydraulics[J]. Journal ofGeological Hazardsand Environment Preservation, 1995, 6(01): 57-64.ZHOU Weiyuan. Advanced Rock Mechanics[M]. Beijing: Water Resources and Electric Power Press, 1993.6, p272-295.

[13] Zheng Shaohe, Zhao Yangsheng, Duan Kanglian. Anexperimental study on the permeability lawof natural fracture under 3-D stresses[J]. Chinese Journal of Rock Mechanics and Engineering, 1999, 18(2): 133-136.

[14] LI Xin-ping, MI Jian, ZHANG Cheng-liang, WANG Yan-li. Analysis of seepage characteristics of rock masses with a single joint under 3D stresses[J]. Rock and Soil Mechanics, 2006, 27(supp.): 13-16.

[15] Chai Jun-rui. Mathematical Model for Coupled Seepage and Stress Fields in Rock Mass of Equivalent Continuum by Considering Hydrodynamic Seepage Pressure[J]. Journal of Sichuan University(Engineering Sciences Edition), 2001, 33(6): 14-17.

[16] Zhang Dianji, Bai Shiwei, Yang Chunhe. Analysis and Study on Permeability of Fractured Rock Mass[J]. Site Investigation Science and Technology, 2003, 01: 24-27.

[17] Liang Bing, Liu Xiaoli, Xue Qiang. Seepage model study of water-rock interaction in low permeability groundwater environment[J]. Chinese Journal of Rock Mechanics and Engineering, 2004,23(5):745-750.

[18] Guo Yulong. Study on the Interaction of the Coupling of Seepage and Stress to Slope stability[D]. Wuhan: Wuhan University of Technology, 2005.

[19] Li Yan, Yang Linde, Yan Xiaobo, Li Peng. Coupling Model and Numerical Simulations on Seepage Flow in Soft Rock[C].The 9th National Conference of Rock Mechanics and Engineering, 2006.

[20] ZHANG Jincail, WANG Jianxue. Coupled behavior of stress and permeability and its engineering applications [J]. Chinese Journal of Rock Mechanics and Engineering, 2006,25(10): 1981-1989.

[21] WANG Yuan, LIU Jie. Complete coupling analysis of transient fluid flow and elastic stress in fractured rock masses $[\mathrm{J}]$. Chinese Journal of Rock Mechanics and Engineering, 2007,26(6):1150-1157. 
[22] LI Yan, YANG Lin-de, DONG Zhi-liang, ZHANG Gong-xin. Experimental research on characteristic of deformation and hydro-mechanical coupling of anistropic rock[J]. Rock and Soil Mechanics,2009,30(5):1231-1236.

[23] ZHANG Yong, PANG Yi-hui. Water-Inrush Mechanical Model Based on a Theory of Coupled Stress-Seepage[J]. Journal of China University of Mining \& Technology, 2010, 39(5): 659-664.

[24] JI You-jun, LIU Jian-jun, CHENG Lin-song. Numerical simulation of tunnel excavation considering fluid solid coupling[J]. Rock and Soil Mechanics, 2011,32(4):1229-1233.

[25] LIN Peng, LIU Xiaoli, HU Yu, XU Wenbin, LI Qingbin. Deformation stability analysis of XILUODU arch dam under stress-seepage coupling condition [J]. Chinese Journal of Rock Mechanics and Engineering, 2013, 32(6):1145-1156.

[26] Kelsall,P.C.,Case,J.B.,Chabames,C.R.,Evaluation1 of Excavation-induced Changes in Rock Permeability, Int.J.Rock Mech. Min. Sci. \& Geomech. Abstr.,Vol.21,No.3,1984,p123-135.

[27] Xu Guangxiang. A Study on Interaction between Seepage Flow and Unloading Mechanics and Drainage of Fractured Rock Masses[D]. Chongqing: Chongqing University, 2001.

[28] Liang Ninghui, Liu Xinrong, Ai Wanmin, Bao Tai. Experiment study on the permeability of fractured rock under unloading[J]. CHINA CIVIL ENGINEERING JOURNAL, 2011, 44(1):88-92.

[29] Ai Wanmin. Experimental Study and Numerical Analysis of the Coupling between Seepage Field and Stress Field of Rock Mass with Unloading[D]. Chongqing: Chongqing University, 2005.

[30] WANG Wei, ZHENG Zhi, WANG Rubin, WANG Huanling, XU Weiya. Experimental study of permeability properties of granitic gneiss under differentstress paths[J]. Chinese Journal of Rock Mechanics and Engineering,2016, 35(2):260-267. 